Pacific Northwest

National Laboratory

Operated by Battelle for the

U.S. Department of Energy

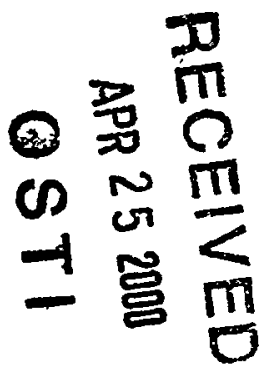

\title{
APMS SVD Methodology and Implementation
}

Brett G. Amidan

Thomas A. Ferryman

March, 2000

Prepared for the U.S. Department of Energy

under contract DE-AC06-76RLO 1831

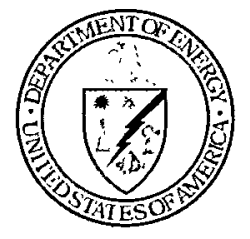




\section{LEGAL NOTICE}

This report was prepared by Battelie Memorial Institute (Battelle) as an account of sponsored research activities. Neither Client nor Battelle nor any person acting on behalf of either:

MAKES ANY WARRANTY OR REPRESENTATION, EXPRESS OR IMPLIED, with respect to the accuracy, completeness, or usefulness of the information contained in this report, or that the use of any information; apparatus, process, or composition disclosed in this report may not infringe privately owned rights; or

Assumes any liabilities with respect to the use of, or for damages resulting from the use of, any information, apparatus, process, or composition disclosed in this report.

Reference herein to any specific commercial product, process, or service by trade name, trademark, manufacturer, or otherwise, does not necessarily constitute or imply its endorsement, recommendation, or favoring by Battelle. The views and opinions of authors expressed herein do not necessarily state or reflect those of Battelle:

This document was printed on recycled paper.

(9/97) 


\section{DISCLAIMER}

This report was prepared as an account of work sponsored by an agency of the United States Government. Neither the United States Government nor any agency thereof, nor any of their employees, make any warranty, express or implied, or assumes any legal liability or responsibility for the accuracy, completeness, or usefulness of any information, apparatus, product, or process disclosed, or represents that its use would not infringe privately owned rights. Reference herein to any specific commercial product, process, or service by trade name, trademark, manufacturer, or otherwise does not necessarily constitute or imply its endorsement, recommendation, or favoring by the United States Government or any agency thereof. The views and opinions of authors expressed herein do not necessarily state or reflect those of the United States Government or any agency thereof. 


\section{DISCLAIMER}

Portions of this document may be illegible in electronic image products. Images are produced from the best available original document. 


\section{APMS SVD Methodology and Implementation}

Brett G. Amidan

Thomas A. Ferryman

March, 2000

Prepared for the U.S. Department of Energy under contract DE-AC06-76RLO 1831

Pacific Northwest National Laboratory Richland, Washington 99352 
C... 


\section{Contents}

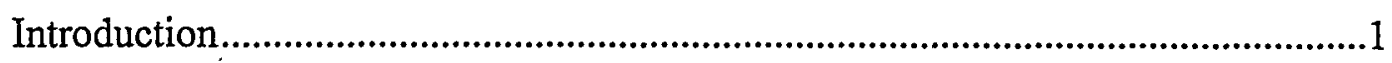

Routine Event Atypicality Methodology .........................................................4

Time Interval Atypicality Methodology ..........................................................

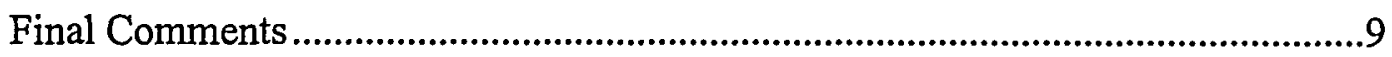

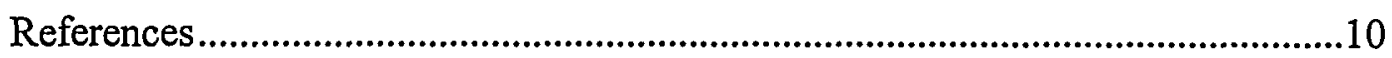

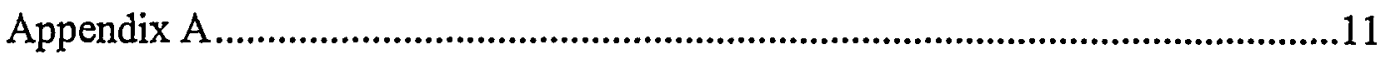

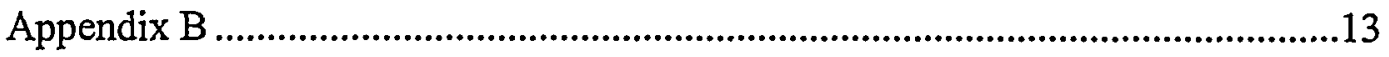




\section{Introduction}

One of the main tasks within the Aviation Performance Measurement System (APMS) program uses statistical methodologies to find atypical flights. With thousands of flights a day and hundreds of parameters being recorded every second for each flight, the amount of data escalates and the ability to find atypical flights becomes more difficult. The purpose of this paper is to explain the method known as single value decomposition (SVD) employed to search for the atypical flights and display useful graphics that facilitate understanding the causes of atypicality for these flights. Other methods could also perform this search and some are planned for future implementation.

The number of parameters for different aircraft ranges from 70 to more than 400 . The data consist of continuous (interval ratio) parameters (that is, airspeed) and categorical (discrete) parameters (such as, air ground switch). Most of the parameters are recorded every second, but some are measured up to eight times a second or once every two seconds. The parameters can be grouped into various parameter sets. These parameter sets contain related parameters, for instance, the Engine parameter set includes engine-related parameters like fuel flow and engine temperature.

These parameter sets can be useful in finding atypical flights. An atypical flight is a flight that, for one reason or another, is different than the other flights. This difference may be obvious, such as an airspeed of $200 \mathrm{mph}$, when all of the other flights have an airspeed close to $150 \mathrm{mph}$. The difference may be more subtle by involving multiple parameters, that by themselves are not atypical, but are when studied collectively. An example of this is a flight that may have an airspeed of $150 \mathrm{mph}$, close to the mean value for that parameter, and a vertical speed of $-2 \mathrm{ft} / \mathrm{sec}$ also close to the average; however, it would be atypical if a flight has both at the same time.

One of the main statistical procedures used to find atypicality is principal component analysis (PCA). This method uses SVD to mathematically transform the variables into eigenvectors. The method is useful in combining the information found in many variables into just a few transformed variables. An example of this process is found in Figure 1. The data for this example consists of 256 records with 16 variables each. The user might choose to investigate the nature of the data by plotting the records using variable pairs as the axes. Plots 1 through 4 show four pairs of these variables being plotted against each other. The relationship that records have to one another cannot be seen clearly in any of these plots. Figure 2 shows a plot of the records using the first two principal components from the PCA of the original data. This plot shows a clear pattern that could not be found in the previous plots because of the multivariate nature of the data. In this case, PCA is able to make order out of what appears to be chaos. The next section will present further detail on how PCA is used to help identify atypical flights. 

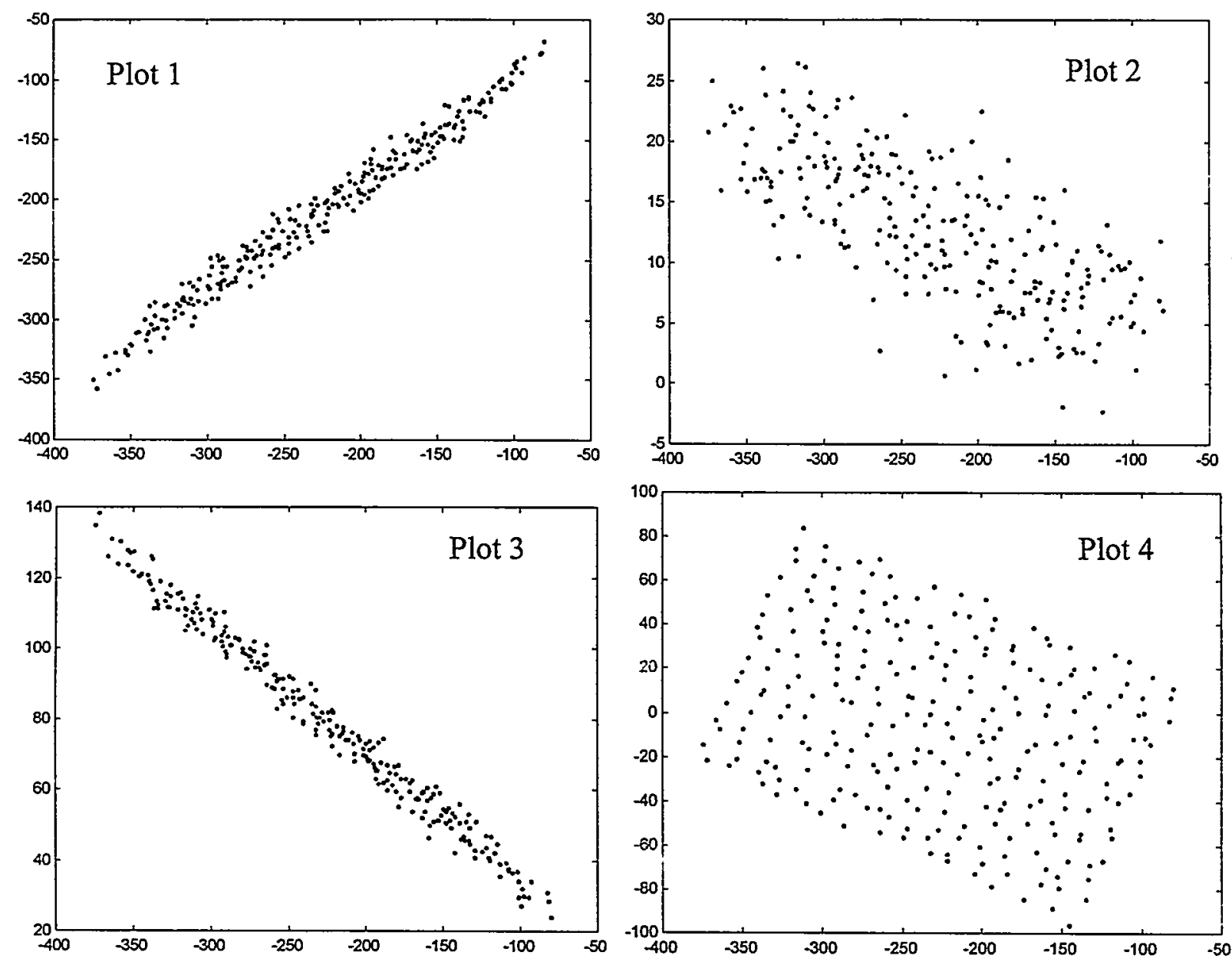

Figure 1. Plots Showing Various Data Presentations of the Multivariate Test Data.

It is common practice for aviation experts to find and investigate atypical flights. Without some help from a statistical analysis they will look at hundreds of typical flights for every flight they find that is atypical. This process can be time-consuming. Furthermore, without the aid of statistical analysis they must rely on their own expertise and perceptiveness. They must compare the data to their own standards of acceptable performance to determine if a flight is atypical or displays a problem. The use of statistical and mathematical methods presents the expert with a set of flights that are much more likely to have noteworthy flight data to study and focuses the attention of the expert on the phase and parameter(s) that are most likely to offer insight to aviation safety issues. The enrichment of the set of flights to look at and the part of each flight to investigate may make the time spent hundreds (or even thousands) of times more effective. Additionally, when a flight is deemed atypical by the software, but the expert does not immediately identify what is significant about that flight phase, this tool will focus their attention 


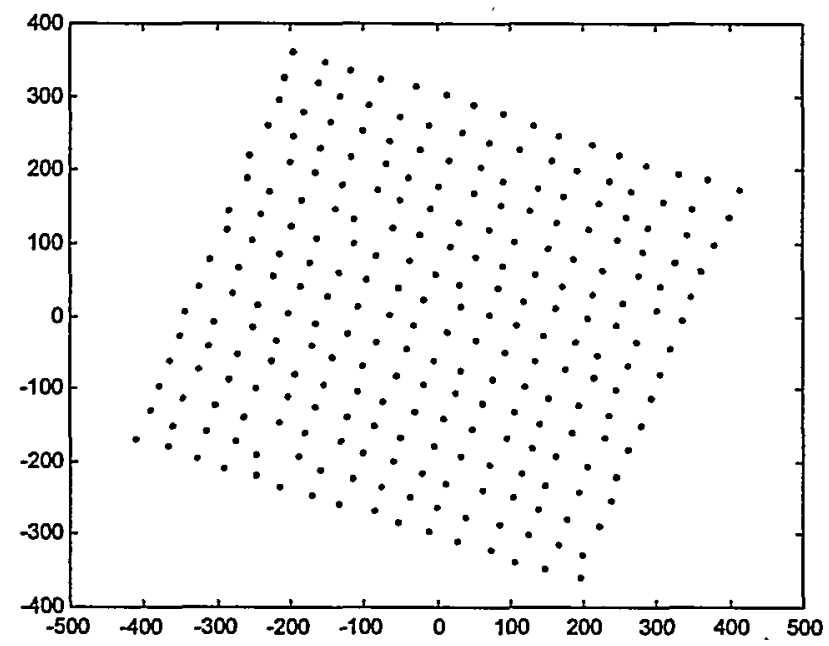

Figure 2. Plot Showing the Nature of the Data from Figure 1.

to atypicality and the parameters that are the most likely contributors. In turn, the tool will inspire the expert to think outside the box and to find more obscure insights that have been hidden in the data and that are necessary to identify to move aviation safety to a higher level.

The first method used to find atypicalities is based on selecting a set of flights that are thought similar to each other in the sense that they should be similar because they have fundamentally common characteristics such as same aircraft type, or same airport/runway (takeoff or landing phases). Then the set of data cut two different ways is used.

The first cut is for analyzing only the snapshots at important events during a flight. Each snapshot is called a routine event (RE). Each RE consists of a static moment of all the appropriate parameters for a given flight at a given event. REs are events like liftoff, 1000 feet height above touchdown, and touchdown. Only those atypicalities that are manifested at an RE are discovered.

The other cut is time interval (TI). This consists of all of the seconds of data that occur within a given phase of a flight. Each flight has been partitioned into five temporal phases. These phases are then subdivided into subphases. These phases and subphases have been designed to allow for the analysis of a time period during a flight that is more homogenous. Examples of these phases and subphases include: liftoff until landing gear up, landing gear up until 10,000 feet above takeoff, cruise, and 1,500 feet above touchdown until 500 feet above touchdown.

Each of the above analysis approaches are discussed in more detail in the next session. 


\section{Routine Event Atypicality Methodology}

As explained in the introduction, the RE data consists of snapshots of the parameter data taken during defined events of a flight. Usually each flight will go through each RE. Analysis of $\mathrm{RE}$ data is limited because it does not consider all the data that occurs between each RE. It relies on finding atypicalities at the most important times of a flight, such as liftoff and touchdown. However, RE analysis is particularly useful in its simplicity, ease of explanation, and the incontestable nature of its findings.

An atypicality score is calculated, using statistical and mathematical methods, for each flight for each individual RE. This process is explained briefly here and a technical explanation is presented in Appendix A. For more details about the methods used, see Rencher 1998. Before any analyses are performed, the data from the RE of interest are standardized (scaled to have a mean of 0 and a standard deviation of 1 ) for each parameter, across all flights. This standardization is needed because many of the parameters are measured in different units and different magnitudes. Therefore, to eliminate a scaling effect, each parameter must be measured in a similar scale, with the standard normal scale as one that is reasonably easy to use and understand.

Next, PCA takes the original parameters and transforms them into linear combinations. This reduces the dimensions of the data from many parameters (that is, 40 or more) to only a few transformed parameters called SVD components (usually 2 or more, depending on the correlation structures in the original data). This reduction is accomplished by finding eigenvalues and corresponding eigenvectors for the data, then ordering the eigenvalues from largest to smallest. Each eigenvector is then multiplied by the original data to create a SVD component. The number of SVD components is the same as that of the variables; however, the first components contain much more information than the last components. Only the components where eigenvalues (starting from largest to smallest) sum to $90 \%$ of the total of all the eigenvalues are kept. These eigenvectors represent $90 \%$ of the variance observed in the data. The other data are thought to represent random variability in the data and no loss of information occurs when they are discarded.

The SVD components can now be plotted against each other in either two or three dimensions. These plots may show outliers in the data, or possible grouping among the data. Figure 3 shows an example of a 2-dimensional scatterplot of two SVD components. Data points can be seen that are far from the pack of data, indicating those flights are atypical from the others.

From the SVD components, an atypicality score is calculated using Mahalanobis distance (McLachlan 1992). The atypicality score is calculated for each flight using the following formula:

$$
\mathrm{A}_{i}=\sum_{j=1}^{n} S V D(j)_{i}^{2} / \lambda_{j}
$$


where $i$ is the flight (row) in which the atypicality score is calculated; $A$ is the atypicality score for a flight; $S V D(j)_{i}$ is the $j$ th SVD component vector and $i$ th element in the vector for $j=1$ (corresponding to largest eigenvalue) to $n$ (corresponding to the smallest eigenvalue not cut-off by the $90 \%$ rule); and $\lambda_{j}$ is the eigenvalue associated with the SVD component of interest. An atypicality score close to zero indicates a typical flight, and a large atypicality score, relative to the other flights, shows an atypical flight.

A histogram of the atypicality scores displays the value for the flights and allows easy identification of atypical flights. Figure 4 shows a histogram of the atypicality scores. The larger the score, the more atypical the flight. In this example, flight $x$ has the largest score and should be the first flight investigated for atypicality. The atypical flights shown in the histogram will generally correspond to the atypical flights seen when plotting each of the SVD components (Figure 3). Histograms can be displayed for any parameter sets or any individual parameters. Histograms of the individual parameters with the atypical flights identified are useful in finding the parameters that are causing the atypicality. Figure 5 shows a histogram of airspeed data (simulated for illustrative purposes, not real data) and shows that flight $x$ is in the tails of the flight distribution and, therefore, a candidate for atypicality.

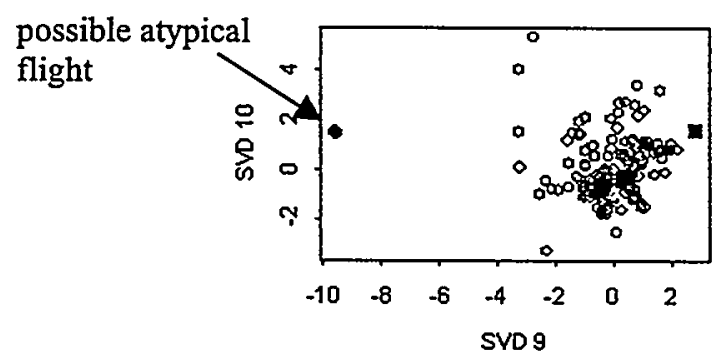

Figure 3. An Example of a 2-Dimensional Scatterplot of Two SVD Components.

Experience has shown that the atypicality histogram (as in Figure 4) has a skewed shape with a long tail to the right. The most atypical flights are far to the right of the typical flights. Atypicalities may be due to one variable being atypical, compared to the other values of that variable for the comparable flights (as in Figure 5). It is possible that several variables are present unusual or subtly unusual values and that they collectively make the flight atypical at this RE.

The APMS tool identifies atypical flights, using comparisons based on all parameters or subsets of parameters. It then displays to the user the flights that are atypical for the selected RE and indicates which parameter(s) are contributing most to the atypicality score, thus showing the aviation expert which flight, which RE, and which parameters to focus on for finding noteworthy observations and generating insightful conclusions from the thousands of flights reviewed by the software. 


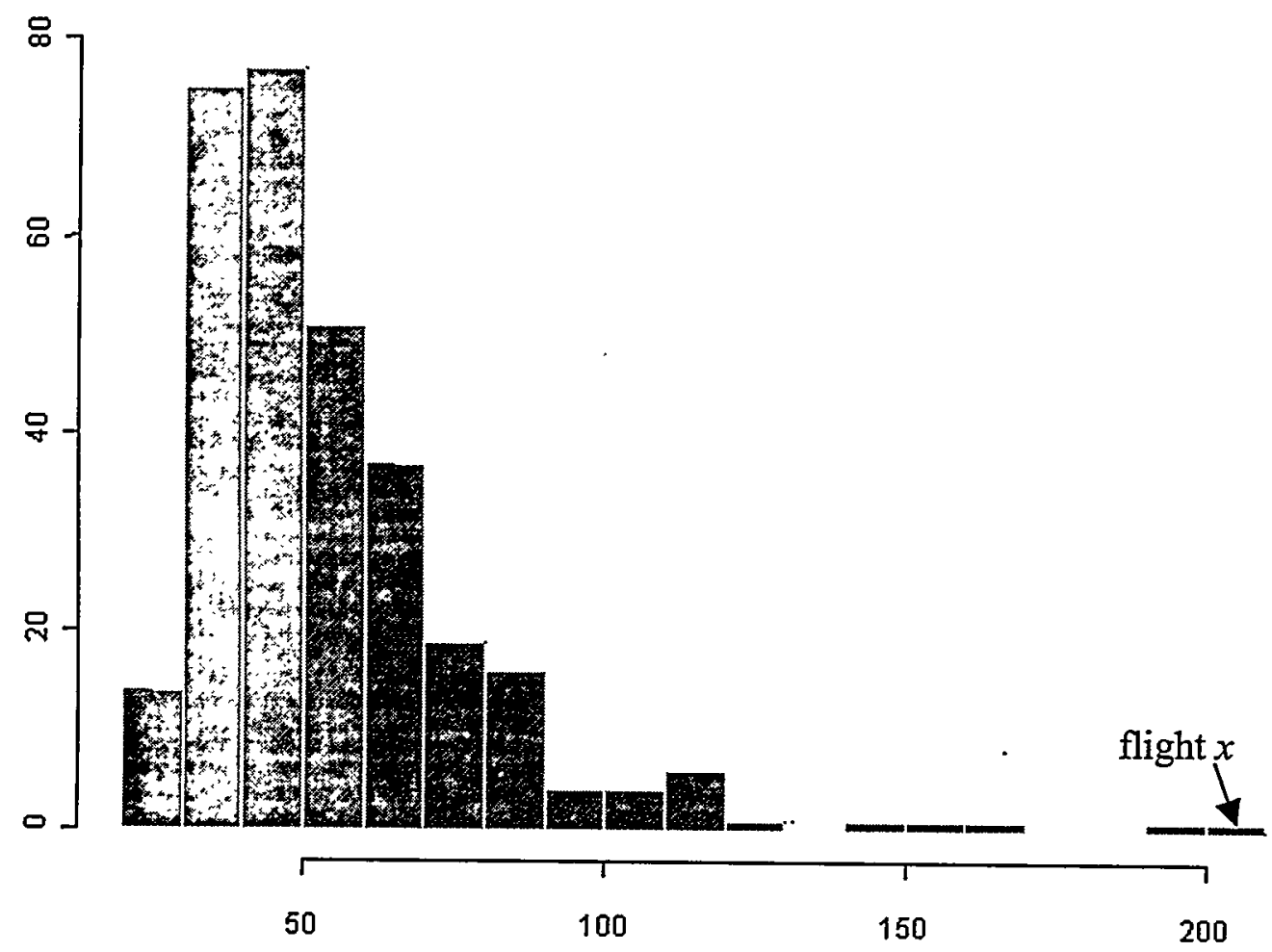

Figure 4. Histogram of Atypicality Scores.

(Atypicality scores on the $\mathrm{x}$-axis, frequency on the $\mathrm{y}$-axis)

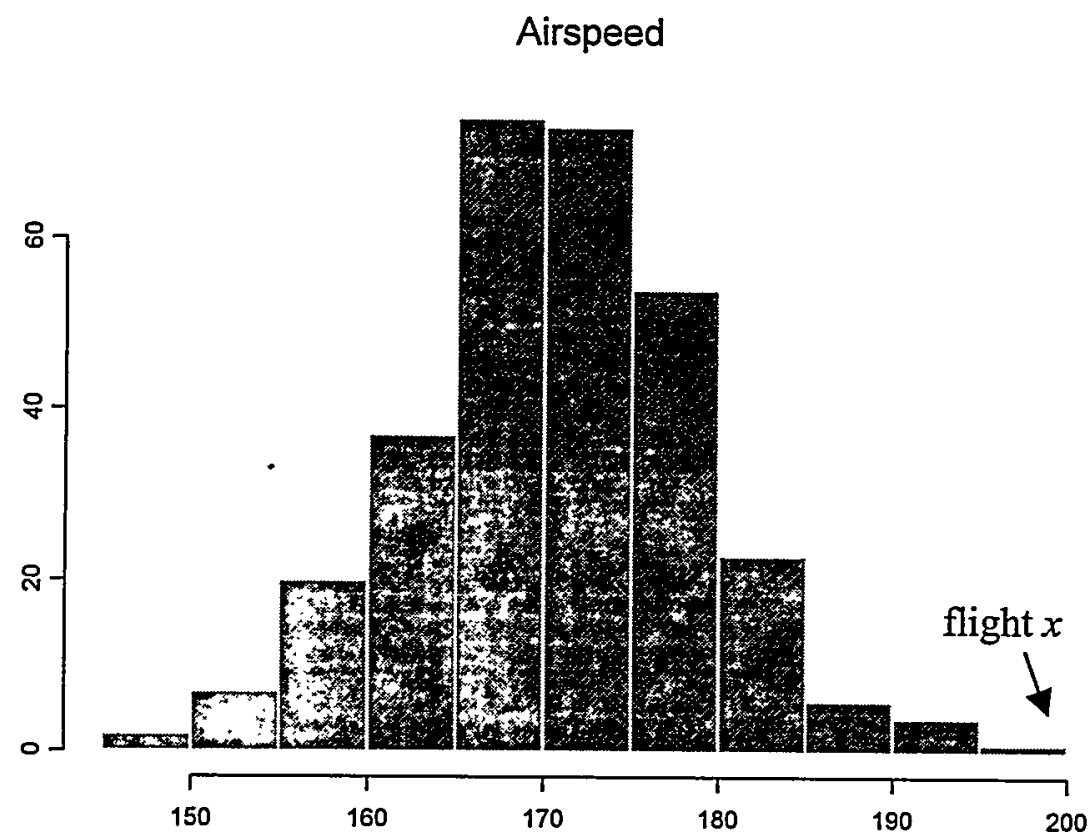

Figure 5. Histogram of Airspeed (not real data). 


\section{Time Interval Atypicality Methodology}

- Time interval (TI) atypicality takes into consideration all the data during a specific time frame, not just a single snapshot. This analysis is valuable because it finds atypicalities that occur between and during the REs, not just during a RE. Because most parameters are recorded every second and flights can last hours, a large amount of data needs to be sifted through: One method to help with this is dividing each flight into five main phases. The five main phases are take-off, ascent, cruise, descent, and touchdown. Each of the five main phases is then subdivided into subphases. These phases and subphases allow the investigator to look across many flights in a time period that is common among the flights. This may show that a flight was atypical at take-off, but typical during the other phases.

Different types of atypicalities may occur during a TI for a given parameter. Obvious atypicalities in a parameter like airspeed may be that one flight's airspeed is consistently higher or lower than the other flights. Another atypicality would be that one flight's airspeed is decreasing during a phase in which all of the other flights' airspeeds are increasing. Still another atypicality may be that a flight's airspeed is fluctuating high and low every few seconds during a phase in which the airspeeds of all of the other flights are constant.

A method to calculate atypicalities has been developed that takes each of these possible atypicalities into consideration across many flights and parameters. A technical explanation of this method can be found in Appendix B. The objective of the TI processing is to summarize each parameter of each flight for each phase or subphase by a mathematical signature. This processing summarizes the many seconds of data for a parameter that occur in a given phase for each flight, providing only a few data statistics that look for the atypicalities mentioned earlier.

The first step in creating this mathematical signature for a given flight and parameter is using 5 seconds of data before and after the data at the first second of the desired phase to create a vector of 11 seconds of data. Then fit a quadratic least squares model, $y=a+b x+c x^{2}$, with time as $x$, the vector of data as $y$, and the coefficients of $a$ (intercept), $b$ (slope), and $c$ (quadratic). An $\varepsilon$ (error) is calculated as a vector of difference between the actual $y$ values and the predicted $y$ values. The $\varepsilon$ vector is converted to a single value, $d=\left[\Sigma \varepsilon^{2}\right]^{1 / 2}$. Repeat this process with the $2^{\text {nd }}$ second of the desired phase and continue to the last second of the same phase. Each second will now have a corresponding set of coefficients $(a, b, c$, and $d)$. Each of the four statistics has a value for each time record in the TI. If the TI is 10,000 seconds long (as cruise might be), 10,000 sets of $a, b, c$ and $d$ 's are calculated. Then each coefficient is summarized by calculating the mean value, standard deviation, minimum value, and maximum value. This calculation results in a mathematical signature for a flight in which the TI data of each parameter is summarized into 16 statistics ( 4 coefficients with 4 statistics each).

To help readers conceptualize this mathematical signature, the following comments are presented. Hopefully, they help to clarify and give examples of what some of the statistics mean. The statistics calculated for the coefficient $a$ represent the magnitude of the parameter values. If a flight has a higher mean $a$ value than the other flights for the parameter airspeed, then its airspeed is on the average higher than the other flights for that given phase. The statistics 
calculated for the coefficient $b$ represent the rate of change (slope) of the parameter values. If a flight has a higher mean $b$ value for the parameter airspeed than the other flights, that means its airspeed is increasing at a higher rate than the other flights for that given phase. The statistics calculated for the coefficient $c$ represent the curvature or rate of rate of change of the parameter values. If a flight has a higher mean $c$ value for the parameter airspeed than the other flights, then its airspeed change in the slope is occurring at a higher rate than the other flights for that given phase. The statistics calculated for the statistic $d$ represent the amount of variability in the parameter values. If a flight has a higher mean $d$ value for the parameter airspeed than the other flights, then that means its airspeed is more variable than the other flights for that given phase. Its airspeed is fluctuating high and low more than the other flights. Other words that conceptually relate to the coefficients $a, b, c$, and $d$ are average value, velocity, acceleration, and noise.

The TI processing results in a data matrix that has $n$ rows and $16^{*} p$ columns, where $n$ is the number of flights (1 row for each flight) and $p$ is the number of parameters (16 statistics for each parameter). Atypicality scores are computed from this data matrix using the same method as in the RE analysis. The only difference between the two is that TI atypicality has 16 times more columns to analyze. Parameter sets (subsets of all of the parameters) can be analyzed, as well as individual coefficients or statistics. For example, if the analyst is concerned about high or low values for the engine parameters, the choice may be to analyze only the engine parameter set and only those columns dealing with coefficient $a$ (the intercept).

As with RE analysis, histograms can show the atypical flights in TI analysis. After a flight has been identified as atypical, the analyst may want to know why it is. Histograms of the mathematical signature data with the atypical flight identified can help zero in on the atypicality. When the parameter(s) with the atypicalities has been identified, then a contour plot is helpful (see Figure 6). This example plot shows altitude above runway versus flap position for Flights 314 and 278. The gray boxes superimposed on the plot display the values of the majority of all other flights; the darker the box, the more flights represented. (This plot does not represent real flight data; it is for illustrative purposes only.) From the plot, Flight 314 looks atypical for flap position because its values are consistently lower than the other flights. It also appears to fluctuate greatly and may be considered atypical for that reason. Flight 278 also looks atypical. 


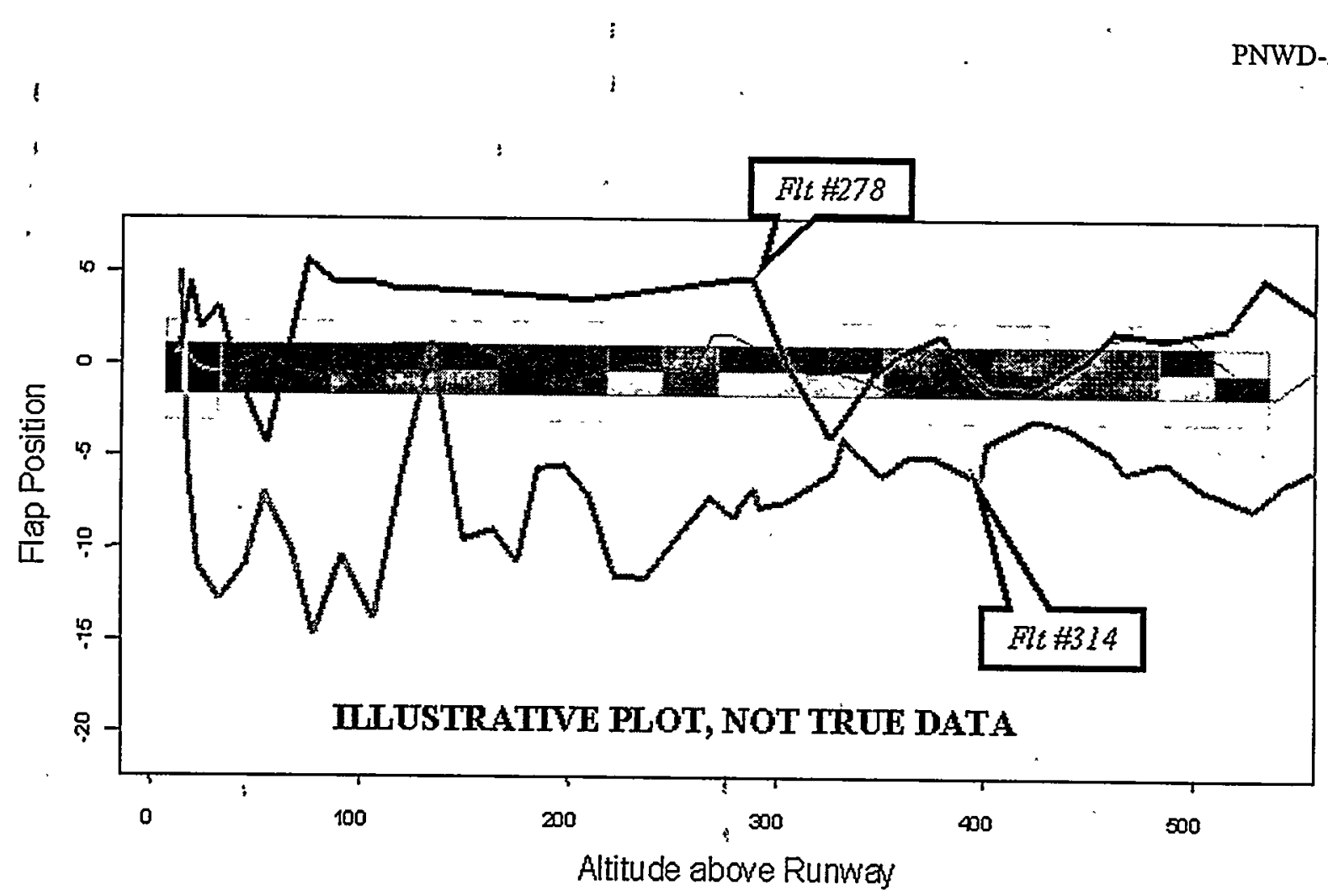

Figure 6. Contour Plot of Flap Position from Take-off until 500 Feet above the Runway.

\section{Final Comments}

SVD has proven to be a viable method to rank flights according to atypicality. With real flight data this method has successfully found interesting flights in a quick and efficient manner. SVD is capable of identifying possible atypical flights but expert experience is required to declare a flight or flight parameter atypical. The user does need to realize that it is very possible that a flight ranked lower in atypicality may actually be more interesting to the expert than the higher ranked flights. For this reason, many flights with higher atypicality scores should be investigated for actual atypicalities. This investigation should be expected because the objective of this SVD method was to calculate atypicalities without any knowledge about aviation safety. Such methodology would allow for an unbiased, purely mathematical approach. Future methods will look at other mathematical and statistical methods and may integrate aviation safety knowledge in order to further filter the atypical flights. 


\section{References}

McLachlan, Geoffrey J. (1992). Discriminant Analysis and Statistical Pattern Recognition. John Wiley and Sons, Inc., New York, NY.

Rencher, Alvin C. (1998). Multivariate Statistical Inference and Applications. John Wiley and Sons, Inc., New York, NY. 


\section{Appendix A: Technical Explanation of the Atypicality Calculations}

Following are the steps used in calculating the atypicality scores:

1. Scale the data $\left(D_{n \times p}\right)$.

$$
D_{c(n \times p)}=\left(\frac{d_{i j}-\bar{d}_{. j}}{\sigma_{j}}\right)
$$

where $d_{i j}$ is the element in the D matrix from row $i$ and column $j, \bar{d}_{j}$ is the average of the $j$ th column from matrix $\mathrm{D}$, and $\sigma_{j}$ is the standard deviation from the $j$ th column from matrix D.

2. Remove all rows of data from $D_{c}$ with at least 1 missing value.

$D_{1((n-m) \times p)}$ is the matrix with no missing values;

$M_{(m \times p)}$ is the matrix with missing values.

3. Calculate the covariance matrix.

$$
C_{(p \times p)}=\operatorname{cov}\left(D_{1}\right)=\left(D_{1}^{\prime} D_{1}\right)^{-1} \sigma^{2}
$$

4. Calculate the eigenvectors and eigenvalues using principle component analysis.

$$
\left[E_{(p \times p)}, F_{(p \times 1)}\right]=\operatorname{Eigen}(C) \quad \text { where } \mathrm{E} \text { is a matrix of eigenvectors and } \mathrm{F} \text { is a vector }
$$

of the eigenvalues.

5. Truncate the number of eigenvectors to use to $q$, where $q$ is the minimal number which satisfies -

$$
\frac{F_{1}+F_{2}+\ldots+F_{q}+F_{q+1}}{\sum F}>\text { threshold. After a threshold is decided upon ( } 0.90 \text { was }
$$

used in these analyses), then $q$ number of eigenvectors and values will be kept.

6. Create the new data. Note: $D_{c}$ is used to create the data, with zeros substituted for the missing values.

$$
G_{c(n x q)}=D_{c(n \times p)} \times E_{(p \times q)}
$$


7. Calculate the Atypicality Scores (Mahalanobis Distance).

$$
A_{i}=\frac{1}{q-m_{i}^{\prime}} \sum_{j=1}^{q} \frac{G_{c(j)_{i}}^{2}}{F_{j}}
$$

where $i$ goes from 1 to $\mathrm{n}$, and $\mathrm{m}^{\prime}{ }_{i}$ is the number of missing values for that row of data. 


\section{Appendix B: Technical Explanation of the Mathematical Signature ${ }^{3}$, Calculations Used in Time Interval Processing}

Following are the steps used in calculating the mathematical signature needed for TI processing:

1. Extract the data for a given flight, given parameter, and given phase. Include the data from 5 seconds before and after the given phase. For example, put data into a vector from Flight \#1, the parameter airspeed during the touchdown phase (including 5 seconds of data before and after the phase). If $m$ is the number of seconds in the given phase, then the extracted data should have $m+10$ seconds.

2. From this extracted data create an 11 -second data window with the first 11 seconds (the 5 seconds before the start of the phase, and the first 6 seconds of the phase).

3. Solve the least squares equation: $y=a+b x+c x^{2}$, with $y$ as the data from the 11-second window, $x$ as the vector $(-5,-4,-3,-2,-1,0,1,2,3,4,5)$, and $a, b, c$ as the coefficients to be solved. l

The coefficient $d$ is calculated using the equation: $d=\sqrt{\frac{S S E}{(n-3)}}$

where $S S E$ is the sums of squares error (from the analysis of variance table), and $n$ is the size of the window (11 in this case).

4. From the data in Step 1, create another 11-second data window by shifting the window over by 1 second, so that seconds 2 through 12 are taken. Then repeat Step 3 with this data and record the coefficients $a, b, c$, and $d$. Continue to take 11-second windows by shifting over 1 second each time, until the last 11 seconds are sampled. This results in a vector of size $m$ for each of the coefficients.

5. Summarize each coefficient vector by calculating the mean, standard deviation, minimum value, and maximum value. This process results in a 16-element vector that summarizes the data for a given flight, given parameter during a given phase. This vector has the following look:

$\bar{a}, \mathrm{~s}_{a}, a_{\min }, a_{\max }, \bar{b}, \mathrm{~s}_{b}, b_{\min }, b_{\max }, \bar{c}, \mathrm{~s}_{c}, c_{\min }, c_{\max }, \bar{d}, \mathrm{~s}_{d}, d_{\min }$, and $d_{\max }$.

6. This process is repeated for each desired flight and each desired parameter and results are put into a matrix with a row for each flight and 16 columns for each parameter (containing the results from each 16-element vector). This matrix is for a given phase.

1. This resulting matrix can be analyzed to find atypical flights within the given phase using'the method explained in Appendix A. 
PNWD-3026

\section{Distribution}

No. of

Copies

OFFSITE

1 I.C. Statler

NASA Ames Research Center MS 262-7

Moffett Field, CA 94035-1000

2 Battelle Mountain View 625 Ellis Street

Suite 305

Mountain View, CA 94043

ATTN: L. Rosenthal

B. Lynch
No. of

Copies

ONSITE

22 Battelle Pacific Northwest Division

B.G. Amidan (10)

K5-12

T.A. Ferryman (2)

$\mathrm{K} 5-12$

A.R. Willse

K5-12

B.A. Pulsipher

K5-12

G.M. Stokes

K9-95

Information Release (7)

K1-06

Distr.1 